\title{
Europa's Alfvén wing: shrinkage and displacement influenced by an induced magnetic field
}

\author{
M. Volwerk ${ }^{1,2}$, K. Khurana ${ }^{3}$, and M. Kivelson ${ }^{3,4}$ \\ ${ }^{1}$ Space Research Institute, Austrian Academy of Sciences, 8042 Graz, Austria \\ ${ }^{2}$ Max-Planck-Institute for Extraterrestrial Physics, 85741 Garching, Germany \\ ${ }^{3}$ Institute of Geophysics and Planetary Physics, UCLA, Los Angeles, CA 90095-1567, USA \\ ${ }^{4}$ Department of Earth and Space Sciences, UCLA, Los Angeles, CA, 90095-1567, USA
}

Received: 31 October 2006 - Revised: 7 March 2007 - Accepted: 30 March 2007 - Published: 8 May 2007

\begin{abstract}
The Galileo magnetometer data are used to investigate the structure of the Alfvén wing during three flybys of Europa. The presence of an induced magnetic field is shown to shrink the cross section of the Alfvén wing and offset it along the direction radial to Jupiter. Both the shrinkage and the offset depend on the strength of the induced field. The entry and exit points of the spacecraft into and out of the Alfvén wings are modeled to determine the angle between the wings and the background magnetic field. Tracing of the Alfvén characteristics in a model magnetic field consisting of Jupiter's background field and an induced field in Europa produces an offset and shrinking of the Alfvén wing consistent with the geometric modeling. Thus we believe that the Alfvén wing properties have been determined correctly. The Alfvén wing angle is directly proportional to the local Alfvén velocity, and is thus a probe for the local plasma density. We show that the inferred plasma density can be understood in terms of the electron density measured by the plasma wave experiment. When Europa is located in the Jovian plasma sheet the derived mass-per-charge exceeds the previous estimates, which is a result of increased pickup of sputtered ions near the moon. The estimated rate of $\mathrm{O}_{2}^{+}$pickup agrees well with the results from numerical models.
\end{abstract}

Keywords. Magnetospheric physics (Current systems; Magnetosphere interaction with satellites and rings; Plasma waves and instabilities)

\section{Introduction}

An Alfvén wing (henceforth AW) is created by the interaction of a conducting body (in this case the moon Europa) with a flowing magnetized plasma. The moon serves as an obstacle in the flow, and will slow the plasma and divert it around

Correspondence to: $\mathrm{M}$. Volwerk

(martin.volwerk@assoc.oeaw.ac.at) its sides. The slowing results from ion pickup and compressional forces. The ion pickup mass-loads the flux tube and creates currents flowing perpendicular to the magnetic field, producing a $\mathbf{j} \times \mathbf{B}$ force (where $\mathbf{j}$ is the electric current and $\mathbf{B}$ is the magnetic field) that decelerates the flow upstream. North and south of the moon along the magnetic field, where the pickup is negligible, the flux tube can propagate unimpeded. This creates a velocity shear along the flux tube bending it. The bend is transported along the magnetic field by an Alfvén wave, with characteristic velocity $v_{\mathrm{A}}=B_{\mathrm{m}} / \sqrt{\mu_{0} \rho}$ where $B_{\mathrm{m}}$ is the unperturbed magnetic field magnitude, $\mu_{0}$ is the permeability of vacuum and $\rho$ is the plasma mass density. In the frame of the conducting moon, the plasma flows across $\mathbf{B}$ at the flow velocity $v_{\text {flow }}$. This will give rise to fronts moving away from the moon at an angle $\theta_{\mathrm{A}}$ with respect to the background magnetic field, which is related to the Alfvén Mach number

$M_{\mathrm{A}}=v_{\text {flow }} / v_{\mathrm{A}}$,

by:

$\theta_{\mathrm{A}}=\operatorname{atan}\left(M_{\mathrm{A}}\right)$.

In the Jovian system the AW was first discussed for Io (Goldreich and Lynden-Bell, 1969; Neubauer, 1980; Southwood et al., 1980; Herbert, 1985; Wright and Southwood, 1987; Chust et al., 2005), and the analysis was later expanded to describe the interaction of other Galilean satellites, in particular Europa (Neubauer, 1998, 1999; Khurana and Kivelson, 1999; Volwerk et al., 1999).

The properties of the AWs, such as the AW angle $\theta_{\mathrm{A}}$, can be useful in determining the local plasma density of the surrounding medium (Neubauer, 1980) if no direct measurements are available. Also, the characteristics of the AW can be used to determine whether there is a internal or induced magnetic field in the object creating the wings. The presence of a magnetic field in the conducting body (a tilted dipole, permanent or induced) will reflect itself in the "shrinking"

Published by Copernicus GmbH on behalf of the European Geosciences Union. 
Jovian Coordinate Systems and Rotations
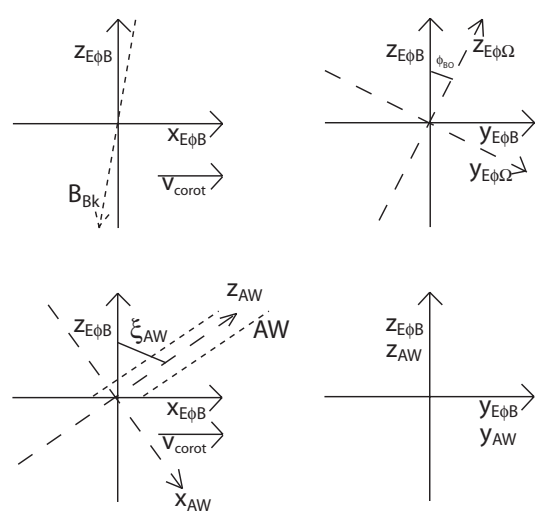

Fig. 1. A schematic overview of the three different coordinate systems in this paper, with projections onto the $x z-, y z-$ and $x y$ plane. Starting from the $\mathrm{E} \phi \mathrm{B}$ coordinate system (solid lines), the top graphs show the definition of the $\mathrm{E} \phi \mathrm{B}$ coordinate system, with the background magnetic field in the $x z$-plane, mainly directed along $z$. A rotation through $\phi_{\mathrm{BO}}$ around the $\mathrm{x}$-axis to obtain the $\mathrm{E} \phi \Omega$ coordinate system (dashed lines). The bottom graphs show how the AW coordinate system (dashed lines) is defined starting from the $\mathrm{E} \phi \mathrm{B}$ coordinate system.

and displacement of the AW (Neubauer, 1999), or an "expansion" if the moon is magnetised with a surface field stronger than the ambient Jovian field, as in the case of Ganymede (Kivelson et al., 1998; Schilling et al., 2003).

\section{Coordinate systems}

In this paper we use three different coordinate systems. Two of them have their x-axes aligned along the corotational flow direction:

\section{1. $\mathbf{E} \phi \mathbf{B}$}

$\mathrm{X}$-axis along the corotational flow direction.

$\mathrm{z}$-axis is chosen such that it is perpendicular to $x$, and the background magnetic field at closest approach is contained in the $x z$-plane.

y-axis completes the triad: $\hat{y}=\hat{z} \times \hat{x}$.

This coordinate system best shows the symmetries related to the magnetic field e.g. the AW.

\section{2. $\mathbf{E} \phi \Omega$}

$\mathrm{X}$-axis along the corotational flow direction.

$\mathrm{z}$-axis is aligned with the Jovian rotation axis.

y-axis completes the triad: $\hat{y}=\hat{z} \times \hat{x}$.

This coordinate system is convenient for discussion of the spacecraft trajectory, because the spacecraft remains close to constant $z$ during a flyby.

We will denote the coordinates and the magnetic field by a subscript " $\mathrm{B}$ " and " $\Omega$ " for $\mathrm{E} \phi \mathrm{B}$ and $\mathrm{E} \phi \Omega$, respectively. The two systems are linked by a simple rotation around the $x$-axis through an angle $\phi_{\mathrm{B} \Omega}$ (given in Table 3) and is visualized in Fig. 1. The cross-section of a cylindrical Alfvén wing is elliptical for a cut at constant $z_{\mathrm{B}}$ and is differently elliptical for a cut at constant $z_{\Omega}$. In Fig. 2 the angle $\alpha$ is labeled in the $\mathrm{E} \phi \mathrm{B}$ system. We do not show the different angles and directions in the $\mathrm{E} \phi \Omega$ system.

The third system that will be used in this paper is specifically related to the AW:

\section{AWS}

z-axis along the AW

$\mathrm{y}$-axis is same as $\mathrm{E} \phi \mathrm{B} \mathrm{y}$-axis

$\mathrm{X}$-axis completes the triad $\hat{x}=\hat{y} \times \hat{z}$

This coordinate system is obtained from the $\mathrm{E} \phi \mathrm{B}$ coordinate system by a rotation around the $y_{\mathrm{B}}$-axis through an angle $\theta_{\mathrm{A}}$ in the Northern Hemisphere or $\pi-\theta_{\mathrm{A}}$ in the Southern Hemisphere. When used in this paper, the ordinates will be labeled "W." The cross-section of a cylindrical AW is circular for constant $z \mathrm{~W}$ if no induced magnetic field is present in the moon, otherwise it is elliptical.

\section{Alfvén wings}

Neubauer $(1998,1999)$ described the AW and its modification by an internal or induced magnetic field (at low Alfvén Mach number). In this section we summarize his analysis.

In Neubauer (1998) the magnetic field that is being transported to Europa is perpendicular to the flow velocity. In the case of Europa, however, the background magnetic field is slightly tilted at a small angle $\theta_{\mathrm{bk}}$ (given in Table 3 ) with respect to the $z_{\mathrm{B}}$-axis. This means that when the AW angle $\theta_{\mathrm{A}}$ is determined, a correction for the slight tilt of the field needs to be made.

The upstream boundaries of the southern portion of the AW are shown in Fig. 2. Note that these fronts represent the locus of the bend in the magnetic field lines and not the direction of the field lines themselves (see e.g. Kivelson et al., 2004, Fig. 1). The bend of the magnetic field lines is produced by currents flowing along the surface of the AW (Neubauer, 1998).

The maximum current flowing along the AW is given by:

$I_{\max }=4 E_{0} R_{\mathrm{W}} \Sigma_{\mathrm{A}}$,

where $E_{0}=v_{\text {flow }} \times B, R_{\mathrm{W}}$ is the radius of the AW and $\Sigma_{\mathrm{A}}$ is the Alfvén conductance $\left(\left(\mu_{0} v_{\mathrm{A}}\right)^{-1}\right.$ Neubauer, 1980). This current flows into the moon on the sub-Jovian side and out of the moon on the anti-Jovian side, and over the whole circumference of the AW the current is described by a $\sin \Phi_{\mathrm{AW}}$ function:

$I\left(\Phi_{\mathrm{AW}}\right)=I_{0} \sin \left(\Phi_{\mathrm{AW}}\right)$,

where $\Phi_{\mathrm{AW}}$ is the angle in the $x y$-plane with respect to the positive $\mathrm{X}$-axis which corresponds to $\Phi_{\mathrm{AW}}=0$. 
This is the simple case for interaction with a nonmagnetized, uniformly conducting moon. However, the presence of an inductive magnetic field in Europa has now unambiguously been shown by Khurana et al. (1998); Kivelson et al. (1999, 2000) and is discussed in great detail by Zimmer et al. (2000).

As noted above, the introduction of an induced magnetic field has two major consequences; the cross-section of the AW shrinks, and its center shifts (Neubauer, 1999). The distortion of the AW depends on the inclination $\alpha_{\text {inc }}$ of the Jovian magnetic field with respect to the Jovian equator at the location of the moon, and the ratio $Q=R_{\mathrm{c}} / R_{\mathrm{A}}$, the ratio of the radius of the conducting body $R_{\mathrm{c}}$ to that of its conducting atmosphere $R_{\mathrm{A}}$. Neubauer shows that the ratio of the radius of the AW to that of the atmosphere $r_{\mathrm{i}}=R_{\mathrm{AW}} / R_{\mathrm{A}}$ depends on $Q$ and $\alpha_{\text {inc }}$ (defined in Fig. $2, \alpha_{\text {inc }}=\pi / 2-\beta_{\text {inc }}$ ). The shape of the AW cross section is not far from circular. The maximum current flowing along the AW is modified by the induced magnetic field:

$$
\begin{aligned}
I_{\max } & \approx 4 E_{0} R_{\mathrm{A}} \Sigma_{\mathrm{A}} r_{\mathrm{i}}\left(\alpha_{\mathrm{inc}}, Q\right) \\
& \approx \frac{4}{\mu_{0}} \sqrt{v_{\text {flow }}^{2} \rho_{0}} R_{\mathrm{A}} r_{\mathrm{i}} .
\end{aligned}
$$

For Europa we can estimate $Q$ and $\alpha_{\text {inc. }}$. For an ionosphere at $H_{\text {ion }}=100-200 \mathrm{~km}$ height (Hall et al., 1998; Kliore et al., $1997)$ and assuming that the conducting layer is located near the surface $\left(1 R_{E}=1560 \mathrm{~km}\right)$ we obtain $Q \approx 0.9$. The minimum inclination of the magnetic field for the three flybys in this paper (E17, E25A and E26) is $\alpha_{\text {inc }} \approx 75^{\circ}$. From Fig. 5 of Neubauer (1999) it follows that we are in the region of $r_{\mathrm{i}} \geq 0.95$, a limit in which there is little distortion of the cross section of the AW provided the Alfvén Mach number is $\ll 1$. The maximum current $I_{\max }$ for the three flybys is given in Table 3.

For Mach numbers relevant to Europa, the cross section of the AW is neither circular, nor elliptical (see Neubauer, 1999, Fig. 4). With a flow velocity $v_{\text {flow }} \approx 105 \mathrm{~km} / \mathrm{s}$, a magnetic field strength $B \approx 400 \mathrm{nT}$ and a plasma density $n_{\mathrm{i}} \approx 100 \mathrm{~cm}^{-3}$ of mass 12.3 (Bagenal, 1994; Kivelson et al., 2004) AMU ions we find that $v_{\mathrm{A}} \approx 250 \mathrm{~km} / \mathrm{s}$ and $M_{\mathrm{A}}=v_{\text {flow }} / v_{\mathrm{A}} \approx 0.4$. In this limit we will approximate the cross-section of the AW by an ellipse.

\section{The data}

To be able to observe the AW near Europa, the spacecraft's orbit needs to pass the moon through a region downstream of the moon that bends away from the background field direction at an angle $\theta_{\mathrm{A}}$. From a total of 12 flybys of Europa, Galileo crossed through the AW on only 3 passes: during the E17 flyby; a non-targeted passage during the I25 orbit, which we will call the E25A flyby; and during the E26 polar flyby. Some parameters of the flybys are given in Table 1; exten-
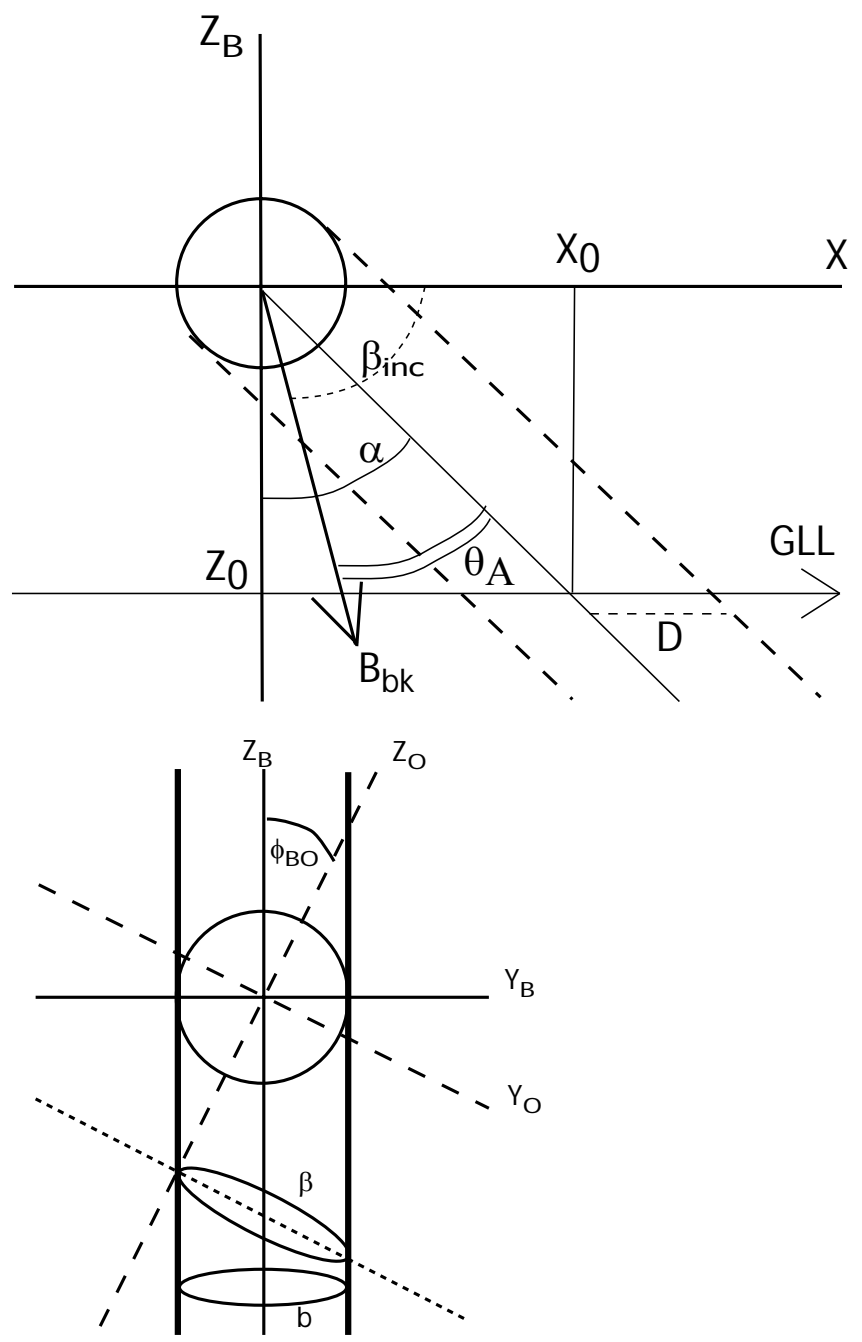

Fig. 2. The topology of the fitted ellipse with semi-major axis $D$ and semi-minor axis $b$. View of the AW in the $x z$-plane. In a simple model of the AW the characteristic moves away from the moon at an angle $\theta_{\mathrm{A}}$ with respect to the background magnetic field $B_{\mathrm{bk}}$ or with an angle $\alpha$ with respect to the $z$-axis. The circle represents Europa and from there the AW is a cylinder with radius $1 R_{E}$. Galileo crosses the AW at $z_{0}$ and the cross-section has the shape of an ellipse whose semi-major axis is defined by $D=z_{0} \tan \alpha$. The inclination of the magnetic field is given by the angle $\beta_{\text {inc }}$.

sive details about the surroundings of Europa in the Jovian magnetosphere can be found in Kivelson et al. (2004).

Figure 3 shows the measured components and the magnitude of the magnetic field across the AWs in the $\mathrm{E} \phi \mathrm{B}$ coordinate system (Kivelson et al., 1992). Clearly visible is the rotation of the magnetic field without a change in total field strength for the first two cases (E17 and E25A). During E26, Galileo passed close to the moon and the signature is influenced by the closure currents in Europa's ionosphere, which both rotate the field and enhance the magnetic field strength. 
Table 1. Flyby overview.

\begin{tabular}{llrrrrrr}
\hline \multirow{2}{*}{ Orbit ID } & \multirow{2}{*}{ date } & DOY & \multicolumn{2}{c}{ Galileo } & \multicolumn{2}{c}{ Europa Sys. III location } \\
& & & time (UT) & altitude $(\mathrm{km})$ & E-long & magn. lat. & LT \\
\hline E17 & 26 Sept 1998 & 269 & $0354: 20$ & 3587.4 & $220^{\circ}$ & $3.6^{\circ}$ & 0955 \\
E25A & 25 Nov 1999 & 329 & $1629: 05$ & 8580 & $166^{\circ}$ & $9.9^{\circ}$ & 0313 \\
E26 & 3 Jan 2000 & 3 & $1859: 43$ & 348.4 & $357^{\circ}$ & $-9.6^{\circ}$ & 0257 \\
\hline
\end{tabular}

The entry into the AW, characterized by the sharp rotation in the magnetic field, takes place over a very short interval, indicating that the Alfvén wing current flows in a region whose thickness is small compared to Europa's radius. In E17, for example, the rotation takes place over 6 data points, i.e. 2 s. Galileo's average velocity with respect to Europa was $8 \mathrm{~km} / \mathrm{s}$ so this corresponds to a thickness of $\sim 16 \mathrm{~km}$. In the discussion section we will return on the specifics of the entry and exit point, e.g. why the entry of E17 is so sharp, whereas the exit seems to be much smoother.

In the $B_{\mathrm{x}}$ components of the data (top panels of Fig. 3) there are significant differences among the three flybys. For E17 a region of strong negative deviation of the magnetic field created by the fringe fields of the currents flowing along the AW is followed by a quick rotation of the field in the $x z$-plane at the entrance into the AW. This occurs at constant magnetic field magnitude as can be seen in the bottom panel of the left column in Fig. 3. For E25A there is little evidence of the fringe fields in $B_{\mathrm{x}}$ before entrance into the AW, however small positive perturbations appear after the AW exit. Note that at E17 Galileo entered the AW at $\Phi_{\mathrm{AW}} \approx-90^{\circ}$, which means at maximum strength of the current in the AW, which explains the strong fringe field, whereas at E25A Galileo entered the AW at a much smaller value of $\Phi_{\mathrm{AW}}$. The negative perturbations of $B_{\mathrm{X}}$ within the AW are expected for a flyby at positive $z_{\mathrm{B}}$. The perturbation magnitude is much smaller than in the E17 case and there is little evidence of the AW in the $B_{\mathrm{Z}}$ component. The E26 data are similar to the E17 data, first a negative deviation because of the fringe fields and then positive perturbation in the AW consitent with a flyby at $z_{\mathrm{B}}<0$. However, the magnetic field magnitude changes because of the effects of closure currents flowing through Europa's surface and ionosphere, as the spacecraft passed much closer to the moon during this flyby (see Table 1). In this paper we do not discuss the $B_{\mathrm{y}}$ component, as this has little influence on our determination of the shape of the AW.

\section{Shrinking of the Alfvén Wing}

Based on the data from the Galileo flybys that intersected the AW, we seek to identify the shrinkage and displacement that are implied by an induced magnetic field (Neubauer, 1999). Athough no analytical expression is given for the shrinkage of the AW, an estimate can be made from first principles. In our model it is assumed that the interior of Europa, where the eddy currents flow that respond to the time varying background magnetic field, is perfectly conducting. This approximation justified by the results obtained by Zimmer et al. (2000) on this conductivity of Europa. It was shown that basically all of the time-varying component of the Jovian magnetic field was cancelled by currents in the moon (see also Schilling et al., 2003). If no induced field is present in Europa, then all field lines in a flux tube of radius $1 R_{E}$ penetrate the moon, giving a magnetic flux $\mathcal{F}=B_{\mathrm{m}} \pi R_{E}^{2}$. However, in the presence of an inductive magnetic field (for a perfect conductor inside the moon) the time varying components of the external magnetic field are cancelled by the moon. In the $\mathrm{E} \phi \Omega$ coordinate system $B_{\mathrm{x}, \Omega}$ and $B_{\mathrm{y}, \Omega}$ are time-varying. In this case we find that the field penetrating into the moon is only $B_{\mathrm{z}, \Omega}$, giving a magnetic flux $\mathcal{F}=B_{\mathrm{z}, \Omega} \pi R_{\mathrm{E}}^{2}$.

Above or below the moon a flux tube that contains field lines that penetrate the moon will be reduced in cross section to a fraction $B_{\mathrm{z}} / B_{\mathrm{m}}$ of the cross section area representing the conducting moon (and its conducting atmosphere). Therefore, the fractional reduction of the cross-section area of the AW can be described by:

$\mathcal{T}=1-\frac{B_{\mathrm{z}, \Omega}}{B_{\mathrm{m}}}$

For Europa $B_{\mathrm{y}, \Omega} \gg B_{\mathrm{x}, \Omega}$ for the unperturbed time varying background field, and thus the shinkage is mainly in the $y-$ direction. If $\gamma_{\mathrm{xy}}=\tan ^{-1}\left(\left|B_{\mathrm{y}} / B_{\mathrm{x}}\right|\right)$, the shrinkage in the $x$ and $y$ direction will be described by $\mathcal{S}_{\mathrm{x}}$ and $\mathcal{S}_{\mathrm{y}}$, which are functions of $\gamma_{\mathrm{xy}}$. We will define these shrinkage factors as follows:

$\mathcal{S}_{\mathrm{x}}=1-\mathcal{T} * \cos ^{2}\left(\gamma_{\mathrm{xy}}\right)$,

$\mathcal{S}_{\mathrm{y}}=1-\mathcal{T} * \sin ^{2}\left(\gamma_{\mathrm{xy}}\right)$,

consistent with the requirement that ratio of the area of the $\mathrm{AW}$ in the presence of induction $\left(A_{\mathrm{AW}}\right.$,ind $)$ to the area when no induction is present $\left(A_{\mathrm{AW}, \mathrm{no}}\right)$, for small angle $\gamma_{\mathrm{xy}}$ is:

$\frac{A_{\mathrm{AW}, \text { ind }}}{A_{\mathrm{AW}, \text { no }}}=\left(1-\mathcal{S}_{\mathrm{x}}\right)\left(1-\mathcal{S}_{\mathrm{y}}\right) \approx 1-\left(\mathcal{S}_{\mathrm{x}}+\mathcal{S}_{\mathrm{y}}\right)=1-\mathcal{T} .(9)$ 
E17

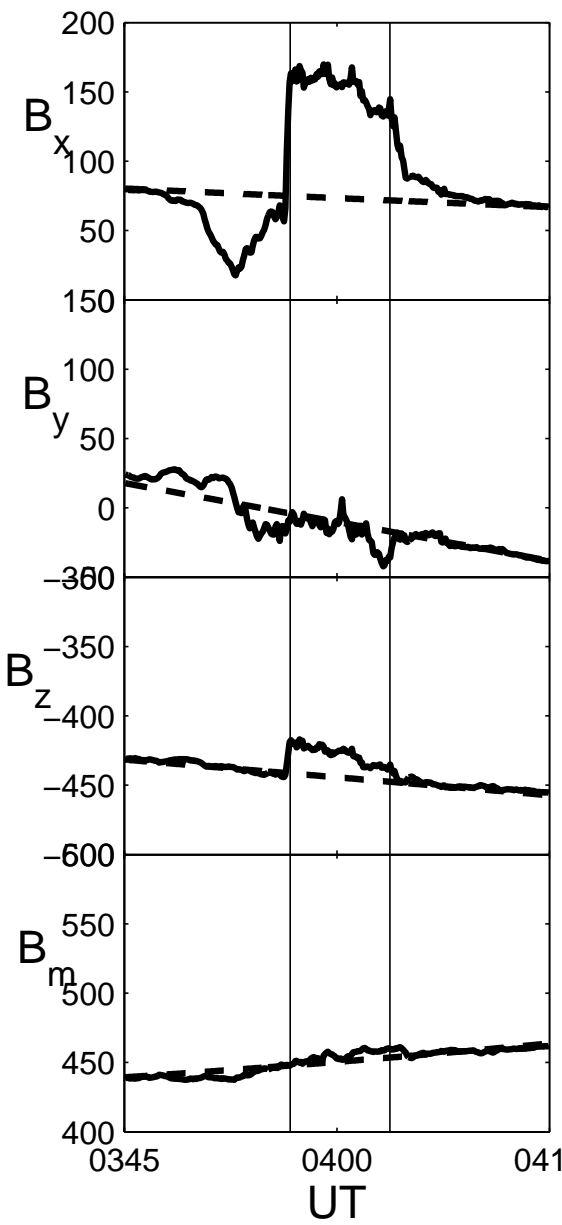

E25A

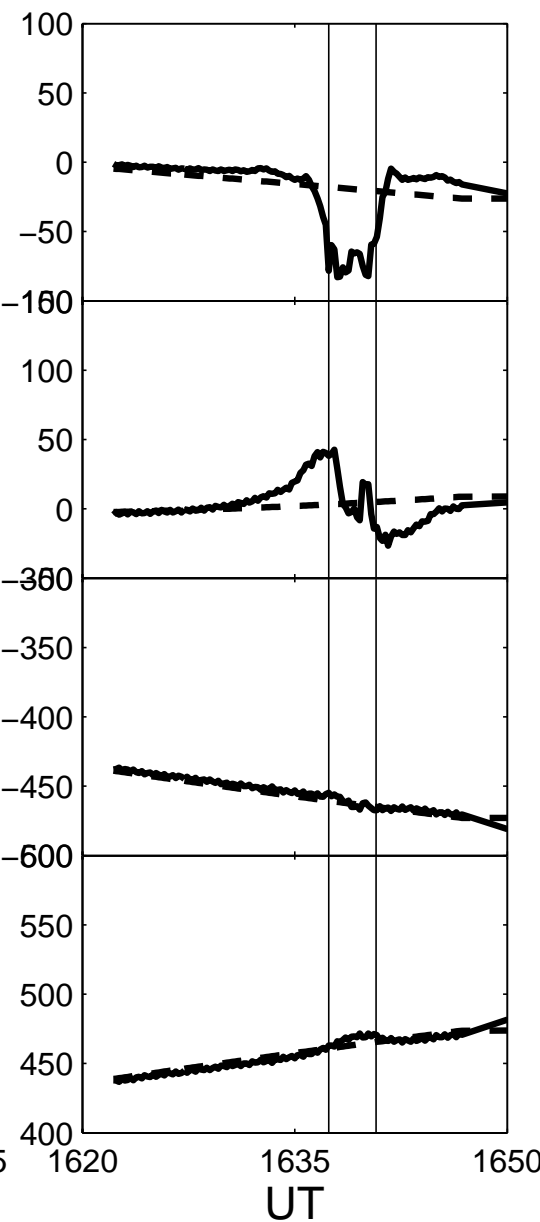

E26

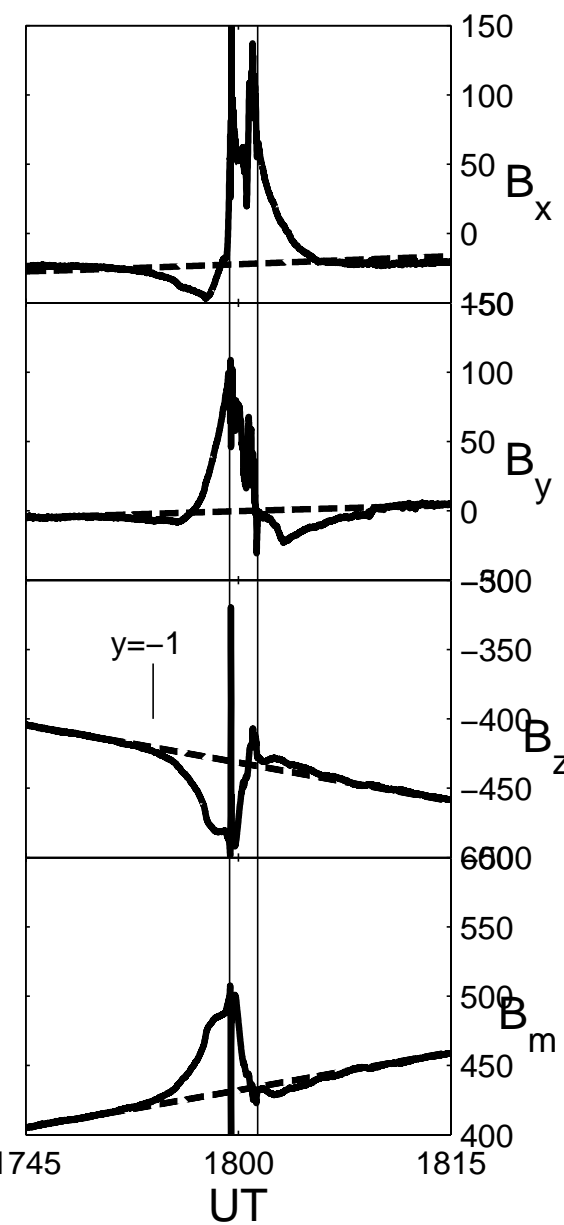

Fig. 3. The magnetometer data of the E17, E25A and E26 flybys in the E $\phi \mathrm{B}$ coordinate system (see text). Shown are the three components of the magnetic field and the magnitude. For E17 and E25A the magnetic field strength remains constant indicating that the interaction currents produce a pure rotation. For E26 the data are influenced by closure current systems near the moon and the field magnitude is affected. The vertical lines show where Galileo enters and exits the AW.

\section{Fitting the Alfvén wing ellipse}

The solution for the parameters of the Alfvén wing can be obtained by transforming the Galileo trajectory to so-called Alfvén wing coordinates, in which the $z_{\mathrm{W}}$-axis is aligned with the appropriate AW characteristic (see Sect. 2). This transformation involves a rotation around the $\mathrm{y}$-axis through an angle $\xi=\theta_{\mathrm{bk}}+\theta_{\mathrm{A}}$ in the $\mathrm{E} \phi \mathrm{B}$ coordinate system (see Figs. 1 and 2). In this coordinate system the cross section at constant $z \mathrm{~W}$ of the unperturbed AW is a circle with a radius of $1 R_{E}$ (or $R_{E}+H_{\text {ion }}$ in case of the presence of an interacting ionosphere of height $H_{\text {ion }}$ ). In the presence of an induced magnetic field, the shape of the AW will be distorted as described above. Note that the shrinking in the y direction (the main component) is invariant under rotation around the y axis. We will use $\mathcal{S}_{\mathrm{x}}$ and $\mathcal{S}_{\mathrm{y}}$ as defined above.
In the new coordinate system the entrance and exit points of Galileo for each flyby are related to the entrance and exit points in the $\mathrm{E} \phi \mathrm{B}$ coordinate systen $\left(x_{0, \mathrm{~B}}, y_{0, \mathrm{~B}}, z_{0, \mathrm{~B}}\right)$ through:

$$
\begin{gathered}
x_{\mathrm{W}}=x_{0, \mathrm{~B}} \cos \xi-z_{0, \mathrm{~B}} \sin \xi, \\
z_{\mathrm{W}}=x_{0, \mathrm{~B}} \sin \xi+z_{0, \mathrm{~B}} \cos \xi,
\end{gathered}
$$

for clockwise rotations over an angle $\xi$ around the y-axis in Fig. 2. In this coordinate system $y_{\mathrm{W}}=y_{0, \mathrm{~B}}$, and the offset of the AW by the induced magnetic field is given by $y_{\text {off. }}$. The initial value of $\theta_{\mathrm{A}}$ is unknown, but can be estimated using the PWS electron density (Gurnett et al., 1992; Kurth et al., 2001) and an assumed average ion mass (see Table 4). After rotation, the equation to be solved for the entrance and the 
Table 2. The Alfvén wing characteristics used in fitting the ellipse and the field line tracing. Given are: the AW entry and exit times of the spacecraft; the coordinates in both $\mathrm{E} \phi \mathrm{B}$ and $\mathrm{E} \phi \Omega$ and the background magnetic field in $\mathrm{E} \phi \mathrm{B}$ and $\mathrm{E} \phi \Omega$.

\begin{tabular}{|c|c|c|c|c|c|c|c|c|c|c|c|c|c|c|}
\hline \multirow[b]{2}{*}{ ID } & & \multirow[b]{2}{*}{$\begin{array}{l}\text { time } \\
\text { UT }\end{array}$} & \multicolumn{6}{|c|}{$\mathrm{E} \phi \mathrm{B}$} & \multicolumn{6}{|c|}{$\mathrm{E} \phi \Omega$} \\
\hline & & & $x_{\mathrm{B}}$ & $\begin{array}{c}y_{\mathrm{B}} \\
R_{\mathrm{E}}\end{array}$ & $z_{\mathrm{B}}$ & $B_{\mathrm{x}, \mathrm{B}}$ & $\begin{array}{c}B_{\mathrm{y}, \mathrm{B}} \\
\mathrm{nT}\end{array}$ & $B_{\mathrm{Z}, \mathrm{B}}$ & $x_{\Omega}$ & $\begin{array}{c}y_{\Omega} \\
R_{\mathrm{E}}\end{array}$ & $z_{\Omega}$ & $B_{\mathrm{X}, \Omega}$ & $\begin{array}{c}B_{\mathrm{y}, \Omega} \\
\mathrm{nT}\end{array}$ & $B_{\mathrm{Z}, \Omega}$ \\
\hline \multirow[t]{2}{*}{ E17 } & in & 0356:42 & 2.06 & -0.9 & -2.47 & 73 & -2 & -431 & 2.06 & -1.42 & -2.21 & 76 & -94 & -429 \\
\hline & out & 0403:45 & 3.29 & 0.15 & -2.18 & & & & 3.29 & -0.33 & -2.16 & & & \\
\hline \multirow[t]{2}{*}{ E25A } & in & $1635: 24$ & 3.28 & -0.86 & 6.25 & -20 & 4 & -464 & 3.28 & 2.55 & 5.76 & -14 & -237 & -385 \\
\hline & out & 1638:44 & 3.42 & 0.40 & 7.01 & & & & 3.42 & 4.03 & 5.75 & & & \\
\hline \multirow[t]{2}{*}{ E26 } & in & 1759:23 & -0.84 & -0.48 & -0.76 & -22 & 0 & -432 & -0.84 & -0.07 & -0.89 & -23 & 208 & -377 \\
\hline & out & 1801:22 & -0.76 & 0.25 & -1.16 & & & & -0.76 & 0.79 & -0.89 & & & \\
\hline
\end{tabular}

Table 3. More encounter parameters: The angle $\phi_{\mathrm{BO}}$ through which the $\mathrm{E} \phi \mathrm{B}$ coordinate system is rotated around the $\mathrm{y}$-axis to obtain the $\mathrm{E} \phi \Omega$ coordinate system; the angle $\theta_{\mathrm{bk}}$ of the magnetic field with the $z_{\mathrm{B}}$ axis; the size of the interacting body $R_{\mathrm{A}}$ (moon radius plus ionosphere) and the maximum current $I_{\max }$ flowing along the AW. The value of $r_{i}$ in Eq. (5) can be taken as 0.95 for these encounters (see Neubauer, 1999, Fig. 5). The values for $v_{0}=90 \mathrm{~km} / \mathrm{s}$ and $\rho_{0}=2500 \mathrm{AMU} / \mathrm{cm}^{3}$ are taken from Kivelson et al. (2004).

\begin{tabular}{lllll}
\hline ID & $\begin{array}{l}\phi_{\mathrm{B} \Omega} \\
\circ\end{array}$ & $\begin{array}{l}\theta_{\mathrm{bk}} \\
\circ\end{array}$ & $\begin{array}{l}R_{\mathrm{A}} \\
\mathrm{km}\end{array}$ & $\begin{array}{l}I_{\max } \\
\mathrm{MA}\end{array}$ \\
\hline E17 & -12 & -9.6 & $1560-1710$ & $0.89-0.95$ \\
E25A & 32 & 2.5 & $1560-1710$ & $0.89-0.95$ \\
E26 & 29 & 2.9 & $1560-1710$ & $0.89-0.95$ \\
\hline
\end{tabular}

exit points of an offset and shrunk circle is:

$$
\frac{x_{\mathrm{W}}^{2}}{\mathcal{S}_{\mathrm{x}}^{2}}+\frac{\left(y_{\mathrm{B}}-y_{\mathrm{off}}\right)^{2}}{\mathcal{S}_{\mathrm{y}}^{2}}=1
$$

or after reorganizing to obtain the offset:

$y_{\text {off }}=y_{\mathrm{B}} \pm \sqrt{1-x_{\mathrm{w}}^{2} \frac{\mathcal{S}_{\mathrm{x}}^{2}}{\mathcal{S}_{\mathrm{y}}^{2}}}$,

where it should be noted that in the case of an interacting ionosphere the 1 in the square root should be replaced with $1+H_{\text {ion }} / R_{\mathrm{E}}$. Only for the correct choice of $\theta_{\mathrm{A}}$ (alternatively $\xi$ in Eq. (13) after Eqs. (10) and (11) have been substituted) is the value of $y_{\text {off }}$ the same for the entrance and exit points. We let $\xi$ vary to obtain the intersection of the two curves describing the offset $y_{\text {off }}$ for the entrance and exit point. The graphical result of this method is shown in Fig. 4. The results for this fitting procedure can also be found in Table 4 , where we show $y_{\text {off }}$ and assuming a charge-per-ion, $Z=1.5$ (Bagenal, 1994) we give the value for the ion mass-per-charge $A / Z$. As a comparison we show the only published PLS plasma density data near Europa (Paterson et al., 1999) for the E4 and E6 flyby.

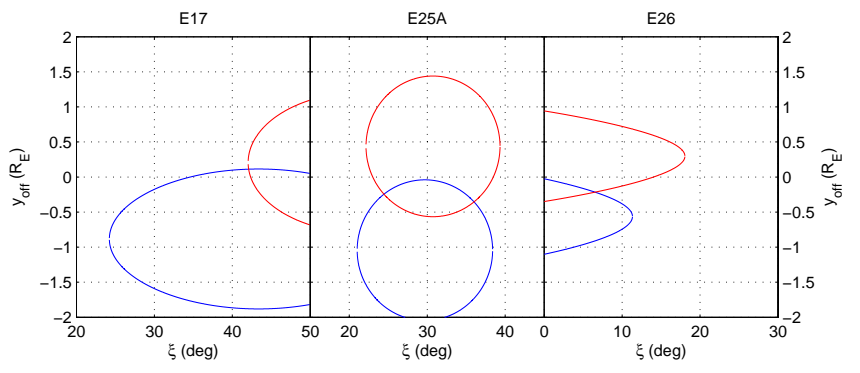

Fig. 4. The curves showing the dependence of $y_{\text {off }}$ on $\xi$ as given in Eqs. (10) and (13) for E17 (left), E25A (middle) and E26. Where the curves for the entry and exit points intersect, the values of both unknowns are found. For the E25A flyby the intersection at $\xi \approx 24$ is the physically correct solution as the second solution $\xi \approx 36$ leads to an unrealistic mass density $(N A>4300)$ for Europa away from the centre of Jovian plasma sheet.

The value obtained for $\theta_{\mathrm{A}}=\xi-\theta_{\mathrm{bk}}$ for each of the flybys can be used to estimate the local plasma density $N A$, assuming that the plasma is at full corotation near Europa with $v_{\text {flow }} \approx 105 \mathrm{~km} / \mathrm{s}$. The Alfvén velocity is obtained from Eq. (1). One finds that:

$$
\begin{aligned}
N A & =\left(\frac{21.8 B_{\mathrm{m}}[\mathrm{nT}]}{v_{\mathrm{A}}[\mathrm{km} / \mathrm{s}]}\right)^{2}, \\
& =0.043 B_{\mathrm{m}}^{2}[\mathrm{nT}] \tan ^{2} \theta_{\mathrm{A}} \mathrm{cm}^{-3} .
\end{aligned}
$$

Inserting the values for $B_{\mathrm{m}}$ and $\theta_{\mathrm{A}}$ from Table 2 we obtain the mass density $N A$ from Eq. (14), which we present in Table 4. We compare the numbers from the Bagenal (1994) torus model $\rho_{\mathrm{m}}$ (see also Kivelson et al., 2004), for the region near Europa with our results. The mass density $N A$ agrees well with the ranges given for $\rho_{\mathrm{m}}$, with E17 slightly exceeding the maximum quoted value (Kivelson et al., 2004). We use the electron density (Kurth et al., 2001), determined from the upper hybrid frequency provided by the Plasma Wave System (PWS) (Gurnett et al., 1992), and a charge-per-ion of 1.5 (Bagenal, 1994; Kivelson et al., 2004) to calculate the average ion mass. The results can be found in Table 4 . We 
find that our ion mass per charge, $10.0 \leq A / Z \leq 17.3$, differs significantly from the value in the literature, $\langle A\rangle / Z=12.3$. We will return to this matter later.

We have performed a similar fitting of the AW for the case of a conducting interacting ionosphere around Europa with $H_{\text {ion }}=150 \mathrm{~km}$. The results are shown in Table 4, where in the column "fit $R_{\mathrm{A}}=1.0 / 1.1$ " the values on the right-hand side of the left slash should be read. As one would expect, for a larger object in the flow the angle $\xi$ decreases for E17 which is at the "downstream" side of the moon, whereas $\xi$ increases for E26 which is at the "upstream" side. The determined offset of the AW $y_{\text {off }}$ naturally also changes.

\section{Alfvén characteristics tracing around Europa}

In order to check the results obtained from fitting the AW in the previous section, a characteristics tracing was performed. In the $\mathrm{E} \phi \Omega$ coordinate system, the spacecraft remains at approximately the same $z_{\Omega}$ during the flybys. Therefore, in this coordinate system a plane at $z_{\Omega}=z_{\text {Galileo for each flyby has }}$ been used to trace the AW characteristics back to Europa. The direction of the AW characteristics is given by the vector sum of the plasma flow velocity, $\mathbf{v}_{\text {flow }}=(105,0,0) \mathrm{km} / \mathrm{s}$ and the Alfvén velocity $\mathbf{v}_{\mathrm{A}}$. To calculate the Alfvén velocity, the plasma density $N A$ as given in Table 4 is used. For the three flybys an area of $4 R_{E}$ by $4 R_{E}$ in the $\mathrm{E} \phi \Omega \times y$-plane was divided up into a grid of $0.05 R_{E}$ separated points in the $\mathrm{x}$ - and y-direction. Each point was traced along the AW characteristic and checked whether it reached Europa, or a region of $1.1 R_{E}$ in case of a Europa with a $150 \mathrm{~km}$ ionosphere.

The result of this tracing can be found in Fig. 5, where the locations that reach Europa are coloured red and the locations that pass within $1.1 R_{E}$ are coloured blue. The green line represents Galileo's flyby and the two cyan dots represent the entry and exit points of the AW by the the spacecraft.

Secondly, the moon was traced to the aforementioned $x y$ plane along the AW characteristic, under the assumption that there was no induced magnetic field present in Europa. The black ellipse in Fig. 5 shows the size and the location of the AW in case of no induced magnetic field. It is clear that the red area is shrunk and offset with respect to the black ellipse.

The tracings show that the densities that were determined in the previous section are acceptable. For the E17 flyby the exit point lies slightly out of the blue area, whereas for the other two flybys the entry and exit poinst are located very near the red-blue boundary.

\section{Discussion}

We have used Galileo's magnetometer data from three crossings of Europa's AW to study the influence of an induced magnetic field. The cross-section of the AW is well described by an ellipse, determined by the size of Europa (possibly including an ionsphere), the angle $\theta_{\mathrm{A}}$ by which the AW moves away from the z-axis and the displacement $y_{\text {off }}$ introduced by an induced magnetic moment. Inductive magnetic fields, generated in the moon by the time varying magnetic field of Jupiter, shrink the cross section of the AW and displace it, and must be taken into account. However, in first approximation, the induced fields do not influence the angle $\theta_{\mathrm{A}}$ between the wing and the background field. This means that by measuring the AW angle $\theta_{\mathrm{A}}$ and e.g. knowing the plasma flow velocity one can get an estimate of the local plasma density through Eqs. (1) and (2), without having to take the induced field into account. A more detailed numerical modelling of the AW interaction of Europa is currently underway by Schilling et al. (2006).

\subsection{Magnetic signature of entrance and exit}

There are strong differences in the magnetic signature of the entrance and exit of the AW, see Fig. 3, in between flybys but also comparing entrance and exit of one flyby. Theoretially, crossing a perfect, plane-parallel infinite current sheet, there will be an abrupt turning of the magnetic field when the spacecraft crosses the sheet. However, in the case of an AW there is a different geometry (cylindrical) and the AW surface current, as explained above in Eq. (4) varies over this cylinder as: $I\left(\Phi_{\mathrm{AW}}\right)=I_{0} \sin \left(\Phi_{\mathrm{AW}}\right)$.

In the previous sections we discussed how how the spacecraft crosses the AW (see Fig. 5). The maximum currents will flow at maximum $y$ in Fig. 5 and the least current at maximum $x$. This means that in the case of E17 Galileo enters the AW at the location of maximum current, and exits the AW at the location of minimal current. Indeed, what one can see in the data is that the entrance into the AW is quite abrupt (after crossing a fringe field region) and the exit of the AW is more gradual over a longer time span. Similarly, in E25A the entrance and exit of the AW happens at an estimated $\Phi_{\mathrm{AW}} \approx \pm 45^{\circ}$, i.e. entrance at a location of "intermediate" current, note that the $\Delta B_{\mathrm{x}}$ at entrance and exit are roughly the same, whereas in E17 there is a significant difference.

Before Galileo's entrance into the AW during the E17 flyby there is a significant negative $\Delta B_{\mathrm{X}}$. This so-called fringe field results from the fact that the AW surface current is not an infinite plane, but curved and varying in current strength. This effect can well be modeled with a wire-current model for the AW (see e.g. Schilling et al., 2003). All three crossings of the AW show this phenomenon, some more clearly then others. Recent work by Schilling et al. (2006) will investigates this, and a better insight will be gained on how and where the currents flow along the AW.

\subsection{E26}

It may be noted that the $B_{\mathrm{m}}$ signature for the E26 flyby shows a significant rise of $\sim 50 \mathrm{nT}$ just before entering the AW and quickly drops during the crossing of the AW. The E26 flyby 


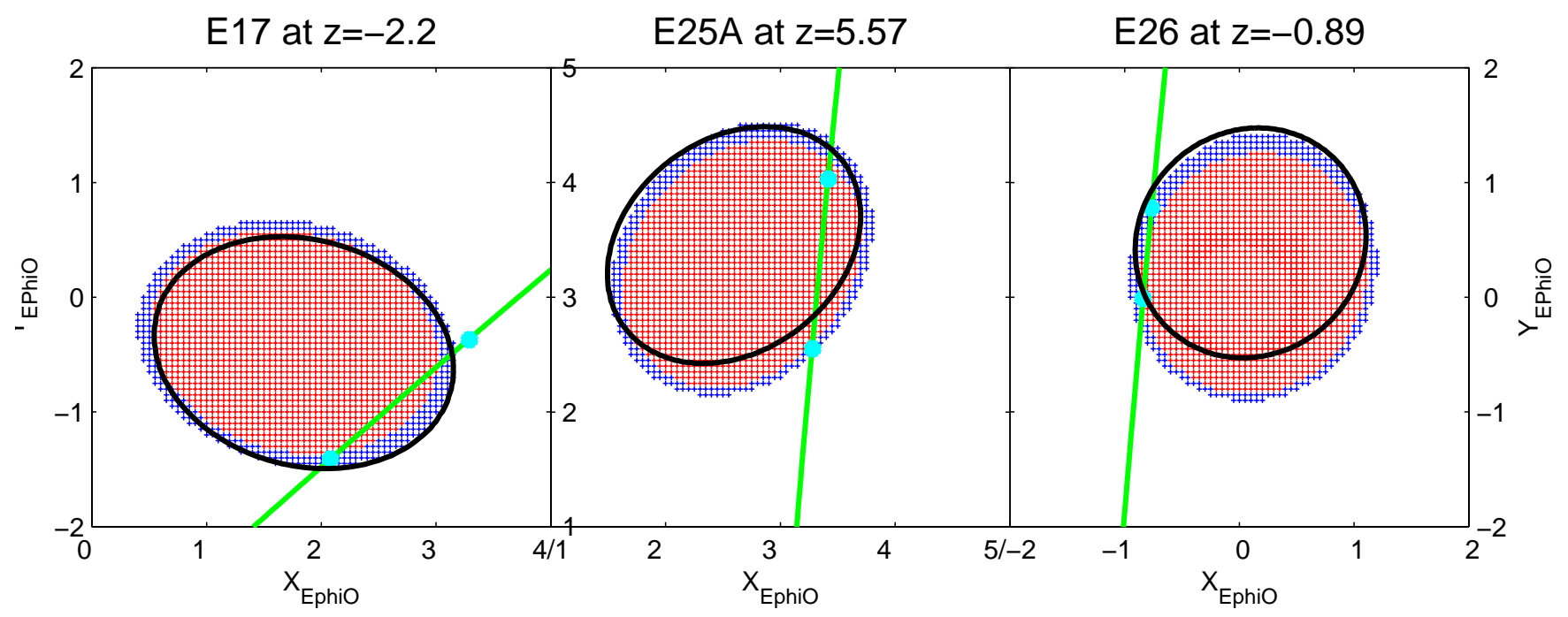

Fig. 5. The cross section of the AW obtained in the E $\phi \Omega x y$-plane by AW characteristic tracings for the E17, E25A and E26 flybys. The red area shows the region which is connected to Europa through the AW characteristic and the blue area shows the region which connects to a possible Europa ionosphere of $150 \mathrm{~km}$. The straight line is Galileo's orbit and the black ellipse is the location of the AW expected if there were no inductive magnetic field present. The big dots on the orbit are the AW entry and exit points observed.

Table 4. The results from the AW fittings: $\mathcal{S}$ (a known parameter), the AW angle $\theta_{\mathrm{A}}$ can be obtained from $\xi$ and the offset $y_{\mathrm{off}}$. The plasma mass densities $N A$ near Europa as determined from the angle $\theta_{\mathrm{A}}$ and Eq. (14), the results are given for two values of the obstacle size, i.e. $1 R_{E}$ and $1 R_{E}+H_{\mathrm{ion}}=1.1 R_{E}$. As a comparison the electron densities $n_{\mathrm{e}}$ are given (Kurth et al., 2001). Nomimal values for the plasma near Europa are: average ion mass $\langle A\rangle=18.5$ AMU/ion, average ion charge $Z=1.5$, (Bagenal, 1994; Kivelson et al., 2004). The PLS ion densities for E4 and E6 have been taken from Paterson et al. (1999).

\begin{tabular}{|c|c|c|c|c|c|c|c|c|c|c|}
\hline \multirow[b]{2}{*}{ ID } & \multirow[b]{2}{*}{$\mathcal{S}$} & \multirow[b]{2}{*}{$\begin{array}{r}|B| \\
\mathrm{nT}\end{array}$} & \multirow[b]{2}{*}{$\begin{array}{r}\rho_{\mathrm{m}} \\
\mathrm{AMU} / \mathrm{cm}^{3}\end{array}$} & \multirow[b]{2}{*}{$\begin{array}{r}n_{\mathrm{e}} \\
\mathrm{cm}^{-3}\end{array}$} & \multicolumn{4}{|c|}{ fit $R_{A}=1.0 / 1.1$} & \multirow{2}{*}{$\begin{array}{r}\text { Lit. } \\
\langle A\rangle / Z \\
\mathrm{AMU}\end{array}$} & \multirow{2}{*}{$\begin{array}{r}\text { PLS } \\
\langle N\rangle(\mathrm{E} 4, \mathrm{E} 6) \\
\mathrm{cm}^{-3}\end{array}$} \\
\hline & & & & & $\begin{array}{l}\xi \\
\circ\end{array}$ & $\begin{array}{r}y_{\text {off }} \\
R_{\mathrm{E}}\end{array}$ & $\begin{array}{r}A / Z \\
\text { AMU }\end{array}$ & $\begin{array}{r}N A \\
\mathrm{AMU} \mathrm{cm}^{-3}\end{array}$ & & \\
\hline E17 & 0.96 & 446 & $200-3000$ & 193 & $42 / 41$ & $0.10 / 0.16$ & $17.8 / 16.5$ & $3463 / 3206$ & 12.3 & $(20,14)$ \\
\hline $\mathrm{E} 25 \mathrm{~A}$ & 0.85 & 452 & $200-3000$ & 70 & $24 / 24$ & $-0.27 /-0.24$ & $14.3 / 14.3$ & $1511 / 1511$ & 12.3 & $(20,14)$ \\
\hline E26 & 0.87 & 431 & $200-3000$ & 20 & $7 / 10$ & $-0.19 /-0.22$ & $10.0 / 18.0$ & $201 / 362$ & 12.3 & $(20,14)$ \\
\hline
\end{tabular}

occurred at very low altitude $(348.4 \mathrm{~km}$, see Table 1$)$, close to Europa's ionosphere. The magnetic signature, therefore, is influenced by the closure currents that flow through the ionosphere and/or through the moon proper and by plasma pickup.

In the Northern Hemisphere the current in the AW flows down on the sub-Jovian side and up on the anti-Jovian side (see Fig. 21.1 in Kivelson et al., 2004). This means that the closure currents flow away from Jupiter. As the flyby took place near the upstream side of Europa (see Fig. 5) we would expect a negative $\Delta B_{\mathrm{Z}}$ produced by the closure currents.

A quick inspection of Galileo's orbit shows that the $B_{Z}$ signature starts to decrease strongly when the spacecraft enters $y \approx-1$, whereas crossing the AW does not occur until $y \approx-0.48$. This means that apart from the closure currents the mass loading near Europa also plays a part in creating a magnetic signature. New pick-up ions will create a current because of the corotational electric field in the Jovian magnetosphere. The current is in the same direction as the closure current, and hence will enhance the magnetic signature. It is quite likely that these pick up currents create the $B_{\mathrm{z}}$ before the spacecraft enters the AW.

Modelling the magnetic signature using a wire current model representing the AW can rather well model the $B_{\mathrm{x}}$ and $B_{\mathrm{y}}$ components, however fail to correctly model the $B_{\mathrm{Z}}$ component (see Fig. 4 in Schilling et al., 2003). The model included the AW and an induced magnetic field (and/or an internal dipole or multipole) and no pick up currents. If it would be possible to include this mass loading to the model the $B_{\mathrm{Z}}$ will most like also be well fitted. Therefore, there is no problem in using the E26 flyby to fit the offset and shrinkage of the AW, even though the flyby sees a significant increase of $B_{\mathrm{m}}$. 


\subsection{Average mass-per-charge}

There is a difference between the derived ion mass-percharge $A / Z$ in this paper and the average value given in the literature (Bagenal, 1994; Kivelson et al., 2004). The difference between our derived ion mass-per-charge and the average ion mass-per-charge in the literature cannot be explained by the assumption that there is sub-corotation of the plasma near Europa. For fixed Alfvén Wing Angle, $\theta_{\mathrm{A}}$ (as determined in Sect. 6, and thus Mach number, $M_{\mathrm{A}}$, sub-corotation (i.e. $v_{\text {flow }}<105 \mathrm{~km} / \mathrm{s}$ ) reduces the inferred the Alfvén velocity (see Eq. 1), $v_{\mathrm{A}}$ using Eq. (14) and hence implies an even larger value of $N A$ and thus $A / Z$. Therefore, our high estimates of $N A$ during the flybys most probably reflect local properties of the plasma near Europa.

The E17 flyby occurs while Europa is exiting the Jovian plasma sheet and sputtering of particles from the moon's surface and pickup is assumed to be at near maximum rate, whereas the E25A and E26 flybys occur when Europa is furthest away from the Jovian plasma sheet and no sputtering takes place. This explains why the ion mass-per-charge inferred for the latter two flybys is close to the average ion mass per charge 14.3 and 10.0 vs. 12.3 . With increased sputtering and pickup near the moon, and the main pickup ion being $\mathrm{O}_{2}^{+}$ with $A=32$ the increased ion mass per charge of 17.3 is easily explained. Volwerk et al. (2001) have shown that for two flybys through Europa's wake that the pickup rate of new ions is significantly higher when Europa is in the Jovian current sheet (E15) compared with when Europa is outside (E11). It was shown that the mass loading rate of $\mathrm{O}_{2}^{+}$ions was almost 3 times as high for E15 compared with E11. Indeed, Saur et al. (1998) have modelled the molecular oxygen loss at a rate of $8.5 \times 10^{26} \mathrm{~s}^{-1}$, for Europa in the Jovian current sheet. However, this loss is dominated by neutral molecular oxygen, and the pick-up loss rate of $O_{2}^{+}$is $1.2 \times 10^{26} \mathrm{~s}^{-1} \mathrm{Using}$ the current results for E17, an estimate can be made of the amount of $\mathrm{O}_{2}^{+}$that is needed to increase the $A / Z$. The electron density is known, $N=193 \mathrm{~cm}^{-3}$, assume that a density $\mathcal{O}$ is molecular oxygen and the rest is the usual plasma with $A / Z=12.3$. Then the density of molecular oxygen is found by:

$12.3(N-\mathcal{O})+32 \mathcal{O}=17.8 N$,

which leads to $\mathcal{O} \approx 53 \mathrm{~cm}^{-3}$. The molecular oxygen will be transported away from Europa with the flow velocity $(\sim 105 \mathrm{~km} / \mathrm{s})$. Assuming a homogeneuos density of the pickup the total loss rate can be estimated as:

Loss $=\mathcal{O} \times v_{\text {flow }} \times \pi \times R_{E}^{2} \approx 4.3 \times 10^{25}$,

where the pickup area is a lower estimate. This rate is approximately a factor 2 to 3 smaller than the rate calculated numerically by Saur et al. (1998). This difference could be explained by the column density of oxygen in the simulation. Recently, (Smyth and Marconi, 2006) calculated the loss rate of molecular oxygen from Europa at $5-10 \mathrm{~kg} / \mathrm{s}$. The estimated loss rate from Eq. (16) implies $2.3 \mathrm{~kg} / \mathrm{s}$.

\subsection{Comparison with numerical models}

There have been many numerical models describing the interaction of the Jovian magnetosphere with the Galilean satellites. Linker et al. (1988, 1991, 1998) studied the interaction of Io with the plasma torus for two different scenarios, Io is either conducting or magnetized. The former could be used to describe Europa's interaction. In these simulations there is no significant offset or shrinkage found of the Alfvén wing.

Another model discussed the interaction of the Jovian magnetosphere with Europa (Saur et al., 1998). However, this model deals mainly with the dynamics of the atmosphere and ionosphere of Europa and the moon is taken as nonconducting, which means that induction can only take place in the ionosphere, while it has been shown to occur under Europa's icy surface (Zimmer et al., 2000) and an ionosphere only may not be enough to cancel the time-varying component of the Jovian magnetic field. In Fig. 10 (Saur et al., 1998), the location of the Alfvén wing currents are shown as contour plots. The maximum current in the wings occurs slightly downstream of $x=0$, however the figure shows no evidence of offset in the $y$-direction or shrinkage.

\section{Conclusions}

We have investigated the Alfvén Wing (AW) signature in the magnetometer data of three Europa flybys. As expected from theory we find that indeed the AW has shrunk and is offset because of the presence of an induced magnetic field in the moon in accordance with Neubauer $(1998,1999)$. Although the fitting of the AW entrance and exit points has been done by an implicit assumption of the shrinkage $\mathcal{S}$, in an upcoming paper Volwerk et al. $(2007)^{1}$ will show that indeed this approach is justified, the flux tube interacting with Europa is shrunk by such a factor.

Tracing of the Alfvén characteristics near the moon confirms that the values of $\theta_{\mathrm{A}}$ and $y_{\mathrm{off}}$ are consistent with the modified Alfvén wing configureation. The inferred distortions provide indirect support for the presence of an inductive dipole moment in Europa.

An estimate of the average ion mass-per-charge in the vicinity of Europa is made using the so-called AW angle, $\theta_{\mathrm{A}}$, and we find that when Europa is away from the Jovian plasma sheet the estimated mass agrees well with that of published models. However, with Europa in the Jovian plasma sheet the local plasma density is enhanced by sputtering and associated

\footnotetext{
${ }^{1}$ Volwerk, M., Paranicas, C., Kivelson, M. G., and Khurana, K. K.: Europa's interaction with the plasma of the Jovian magnetosphere: The wake region, in preparation, 2007.
} 
ion pickup, leading to a much larger average ion mass-percharge than the standard model of the Jovian plasma sheet.

Acknowledgements. The authors would like to thank B. Kurth for making the E25A density available. Part of the work by M. Volwerk was financially supported by the German Bundesministerium für Bildung und Forschung and the Zentrum fur Luft- und Raumfahrt under contract 50 OC 0103 . This research was supported by the National Aeronautics and Space Administration through Jet Propulsion Laboratory under contract JPL 958694 and under contract NAG 5-7959.

Topical Editor I. A. Daglis thanks two referees for their help in evaluating this paper.

\section{References}

Bagenal, F.: An empirical model of the Io plasma torus: Voyager measurements, J. Geophys. Res., 99, 11 043-11 062, 1994.

Chust, T., Roux, A., Kurth, W. S., Gurnett, D. A., Kivelson, M. G., and Khurana, K. K.: Ar Io's Alfvén wings filamented? Galileo observations, Planet. Space Sci., 54, 395-412, 2005.

Goldreich, P. and Lynden-Bell, D.: Io, a jovian unipolar inductor, Astrophys. J., 156, 59-78, 1969.

Gurnett, D. A., Kurth, W. A., Shaw, R. R., Roux, A., Gendrin, R., Kennel, C. F., Scarf, F. L., and Shawhan, S. D.: The Galileo plasma wave investigation, Space Sci. Rev., 60, 341-355, 1992.

Hall, D. T., Feldman, P. D., McGrath, M. A., and Strobel, D. F.: The far-ultraviolet oxygen airglow of Europa and Ganymede, Astrophys. J., 499, 475-481, 1998.

Herbert, F.: "Alfvén wing" models of the induced electrical current system at Io: A probe of the ionosphere of Io, J. Geophys. Res., 90, 8241-8251, 1985.

Ip, W.-H.: Europa's oxygen exosphere and its magnetospheric interaction, Icarus, 120, 317-325, 1996.

Khurana, K. K., Kivelson, M. G., Stevenson, D. J., Schubert, G., Russell, C. T., Walker, R. J., Joy, S., and Polansky, C.: Induced magnetic fields as evidence for subsurface oceans in Europa and Callisto, Nature, 395, 777-780, 1998.

Khurana, K. K. and Kivelson, M. G.: Inner Jovian magnetosphere: mass-loading near Europa, EOS, 80, F875, 1999.

Kivelson, M. G., Khurana, K. K., Means, J. D., Russell, C. T., and Snare, R. C.: The Galileo magnetic field investigation, Space Sci. Rev., 60, 357-383, 1992.

Kivelson, M. G., Warnecke, J., Bennett, L., Joy, S., Khurna, K. K., Linker, J. A., Russell, C. T., Walker, R. J., and Polanskey, C.: Ganymede's magnetosphere: Magnetometer overview, J. Geophys. Res., 103, 19963-19972, 1998.

Kivelson, M. G., Khurana, K. K., Stevenson, D. J., Bennet, L., Joy, S., Russell, C. T., Walker, R. J., Zimmer, C., and Polanski, C.: Europa and Callisto: Induced or intrinsic fields in a periodically varying plasma environment, J. Geophys. Res., 104, 4609-4625, 1999.

Kivelson, M. G., Khurana, K. K., Russell, C. T., Volwerk, M., Walker, R. J., and Zimmer, C.: Galileo magnetometer measurements strengthen the case for a subsurface ocean at Europa, Science, 289, 1340-1343, 2000.
Kivelson, M. G., Bagenal, F., Kurth, W. S., Neubauer, F. M., Paranicas, C., and Saur, J.: Magnetospheric interactions with satellites, Chapter 21, in: Jupiter: The Planet, Satellites and Magnetosphere, edited by: Bagenal, F., Dowling, T. E., and McKinnon, W. B., 513-536, Cambridge University Press, 2004.

Kliore, A. J., Hinson, D. P., Flasar, F. M., Nagy, A. F., and Cravens, T. E.: The ionosphere of Europa from Galileo radio occultations, Science, 277, 355-358, 1997.

Kurth, W. S., Gurnett, D. A., Persoon, A. M., Roux, A., Bolton, S. J., and Alexander, C. J.: The plasma wave environment of Europa, Planet. Space Sci., 49, 345-363, 2001.

Linker, J. A., Kivelson, M. G., and Walker, R. J.: An MHD simulation of plasma flow past Io: Alfvén and slow mode perturbations, Geophys. Res. Lett., 15, 1311-1314, 1988.

Linker, J. A., Kivelson, M. G., and Walker, R. J.: A threedimensional MHD simulation of plasma flow past Io, J. Geophys. Res., 96, 21 037-21 053, 1991.

Linker, J. A., Khurana, K. K., Kivelson, M. G., and Walker, R. J.: MHD simulations of Io's interaction with the plasma torus, J. Geophys. Res., 103, 19867-19877, 1998.

Neubauer, F. M.: Nonlinear standing Alfvén wave current systen at Io: Theory, J. Geophys. Res., 85, 1171-1178, 1980.

Neubauer, F. M.: The sub-Alfvénic interaction of the Galilean satellites with the Jovian magnetosphere, J. Geophys. Res., 103, 19834-19866, 1998.

Neubauer, F. M.: Alfvén wings and electromagnetic induction in the interiors: Europa and Callisto, J. Geophys. Res., 104, $28671-$ $28684,1999$.

Paterson, W. R., Frank, L. A., and Ackerson, K. L.: Galileo plasma observations at Europa: Ion energy spectra and moments, J. Geophys. Res., 104, 22 779-22 791, 1999.

Saur, J., Strobel, D. F., and Neubauer, F. M.: Interaction of the Jovian magnetosphere with Europa: Constraints on the neutral atmosphere, J. Geophys. Res., 103, 19947-19962, 1998.

Schilling, N., Khurana, K. K., and Kivelson, M. G.: Limits on an intrinsic dipole moment in Europa, J. Geophys. Res., 109, E05006, doi:10.1029/2003JE002166, 2004.

Schilling, N., Neubauer, F. M., and Saur, J.: Interaction of Europa with the Jovian magnetosphere, AGU Fall Meeting Abstracts, F5+, 2006.

Smyth, W. H. and Marconi, M. L.: Europa's atmosphere, gas tori and magnetospheric implications, Icarus, 181, 510-526, 2006.

Southwood, D. J., Kivelson, M. G., Walker, R. J., and Slavin, J. A.: Io and its plasma environment, J. Geophys. Res., 85, 5959-5968, 1980.

Volwerk, M., Kivelson, M. G., and Khurana, K. K.: Europa's Alfvén wing: Skewness and field aligned currents, EOS, 80, F875, 1999.

Volwerk, M., Kivelson, M. G., and Khurana, K. K.: Wave activity in Europa's wake: Implications for ion pick-up, J. Geophys. Res., 106, 26 033-26 048, 2001.

Wright, A. N. and Southwood, D. J.: Stationary Alfvénic structures, J. Geophys. Res., 92, 1167-1175, 1987.

Zimmer, C., Khurana, K. K., and Kivelson, M. G.: Subsurface oceans on Europa and Callisto: Constraints from Galileo magnetometer observations, Icarus, 147, 329-347, 2000. 